\title{
Système médiatique, nationalisme et démocratie en Belgique
}

Interview

Dave Sinardet ${ }^{1}$

Expert en nationalisme et fédéralisme ainsi qu'en communication politique, Dave Sinardet a étudié le rôle des médias dans la construction des identités nationales, notamment dans des pays fédéraux multilingues comme la Belgique. Chroniqueur, commentateur et observateur de la vie politique belge, Dave Sinardet écrit pour de nombreux médias belges, tant néerlandophones que francophones. Il répond aux questions de Julien Danero Iglesias et Marie Fierens concernant le lien qui existe entre les médias et les identités nationales et applique son analyse au contexte particulier de la Belgique.

Quel est le rôle des médias dans la construction des identités en général, et des identités politiques en particulier? Assument-ils un rôle d'acteurs? Ou doivent-ils davantage être considérés comme des instruments?

DS : Les médias restent très importants dans le processus de construction des identités et notamment des identités nationales. Ce rôle a déjà été souligné dans les théories classiques sur le nationalisme. Selon l'anthropologue et politologue Benedict Anderson par exemple, la connaissance de base de la nation et de ses habitants est rendue possible par une série d'instruments socialisateurs, répandus largement, comme les mass-médias. Ces instruments intègrent le plus grand nombre dans la vie «nationale ». De cette façon ces médias contribuent à produire «le» public national, et donc également la « nation » à laquelle ils s'adressent.

Aujourd'hui encore, la plupart des médias populaires sont organisés sur une base nationale, ce qui leur fait jouer ce rôle de construction. Ils s'adressent explicitement au public qui réside à l'intérieur des frontières nationales.

De plus, le contenu de leur offre contribue à construire et entretenir ces identités nationales, comme l'ont montré beaucoup d'études de contenu médiatique portant tant sur des émissions de fiction que d'information. Les émissions d'actualité en particulier contribuent à créer et entretenir un cadre de référence national commun à

1 Vrije Universiteit Brussel. 
travers lequel les informations sont interprétées. Ainsi, les informations étrangères un concept qui est «nation-centrique » en lui-même - sont souvent présentées dans un cadre national, comme lorsque l'attention est focalisée sur les victimes du pays émetteur de l'information, à l'occasion d'une catastrophe à l'étranger. Dans la littérature anglophone, on appelle cela la "domestication" de l'information.

Par ailleurs, l'aspect linguistique, le langage utilisé, est également très important. Par exemple, des expressions comme "notre pays », «chez nous», etc. renvoient au public imaginé de la nation. En même temps, les frontières de la nation sont indiquées par la différence faite entre "nous » et « eux ». Le lecteur ou le téléspectateur ne remarque plus l'utilisation routinière de ce type de langage. Ce processus de routinisation est crucial car il nous rappelle inconsciemment le collectif auquel nous appartenons.

Est-ce une bonne chose ou une mauvaise chose? Le rôle des médias tel que vous venez de le décrire peut-il être évalué de manière positive ou négative?

DS: Cela dépend de la position que l'on prend. Dans tous les cas, ce rôle n'est pas neutre. Il revêt un aspect idéologique, au sens large du terme. Le nationalisme politique considère que la nation devrait coïncider avec l'Etat. Pour cela il faut bien sûr qu'une telle nation existe, notamment dans les esprits, et qu'elle soit considérée comme importante par ceux qui en font partie. Les médias peuvent soutenir ce projet en participant à la construction de cette nation dans l'esprit de leur public. Ils peuvent en effet renforcer les conditions et les prémisses sur lesquelles s'appuie le projet nationaliste. Les médias renforcent l'idée selon laquelle la division du monde en nations est naturelle. En ce sens, ils peuvent être considérés comme des propagateurs de nationalisme, comme le suggère l'historien Ernest Gellner.

Le sociologue britannique Michael Billig parle quant à lui de «nationalisme banal ». Ce concept ne se réfère pas à l'image d'un drapeau national que l'on brandit avec ferveur lors d'une manifestation politique, mais à celle du même drapeau flottant devant un bâtiment officiel sans que l'on y porte plus attention. Il s'agit d'un nationalisme implicite, que l'on retrouve aussi dans les médias, comme je viens de l'illustrer. Ce nationalisme banal donne l'impression que notre appartenance à une nation est naturelle, ce qui n'est évidemment pas le cas. Les nations ne sont pas des phénomènes naturels, mais des constructions sociales créées au gré de circonstances historiques et économiques spécifiques. Ce caractère construit se remarque surtout lorsque, au sein d'un même territoire, différentes conceptions de la nation s'affrontent. Pour certains par exemple, le territoire écossais fait partie de la nation britannique ; pour d'autres, il fait partie de la nation écossaise; pour d'autres encore, il fait partie des deux. C'est la même chose dans le cas du territoire de la région flamande, qui selon certains fait partie d'une nation belge et, selon d'autres, d'une nation flamande, ou encore des deux. 
Face à de tels conflits identitaires, les médias peuvent jouer un rôle plus explicitement idéologique ou politique, en représentant une identité contestée au détriment d'une autre, contribuant ainsi à la naturalisation de la première. Les médias peuvent alors devenir les instruments d'un projet politique de «nation-building ».

Vous avez abordé le cas de la Belgique. Quel est précisément le rôle assumé par les médias belge dans la construction des identités nationales?

DS : En Belgique, les médias sont presque entièrement organisés sur des bases communautaires. Le simple fait qu'il existe des médias flamands et francophones mais qu'il n'existe aucun média belge engendre la construction d'identités sous-étatiques. C'est la forme de construction d'identité nationale la plus banale, et donc probablement aussi la plus efficace.

Lorsque, en 1960, on scinde l'émetteur public unitaire en deux institutions quasi autonomes, c'est, d'un point de vue institutionnel, un pas important vers la défédéralisation culturelle et la création de deux grandes communautés linguistiques. C'est d'ailleurs ce qui s'est passé officiellement dix ans plus tard. En 1970, quand ces dernières ont été créées institutionnellement, elles ont directement obtenu la compétence de réglementer les médias. Si l'on adopte une perspective internationale, on se rend compte que cette division du paysage médiatique belge sur base linguistique est très poussée. Dans des pays comme le Canada ou la Suisse, il y a aussi des chaînes différentes pour les différents groupes linguistiques, mais on retrouve au moins une structure nationale de télévision publique, dont la compétence relève (en partie) du gouvernement national.

On peut donc dire que l'absence de médias nationaux rend difficile la formation d'une identité «belge». Pour autant, est-il possible d'affirmer que les médias francophones/wallons et flamands construisent des identités particulières?

DS : Du côté flamand, dès sa création, la télévision publique s'inscrit dans un projet politique identitaire. Déjà avant la scission institutionnelle, l'élite catholique et flamingante qui dirigeait le service public voyait le nouveau média qu'était la télévision comme un instrument très utile pour l'émancipation culturelle de la Flandre et pour la construction d'une identité flamande, comme le montre l'étude de Hilde Van Den Bulck. Cette élite utilisait donc les médias dans un projet de "nation-building " flamand, sans pour autant être anti-belges. Plus tard, le gouvernement flamand - clairement conscient du rôle que les médias peuvent jouer dans la construction des identités - assigne comme mission à la télévision publique flamande de contribuer au renforcement de l'identité flamande. Son nom évolue aussi de BRT (« Radio et télévision belge ») en BRTN (Radio et télévision belge, émissions néerlandophones) et finalement en VRT (« Radio et télévision flamande»). Dans les années 1990, une circulaire 
a même demandé à la VRT de ne plus parler de la « côte belge », mais de la « côte flamande ».

Ce qui est paradoxal, c'est qu'en dépit de ces multiples tentatives, toutes les études scientifiques portant sur les identités montrent qu'en Flandre, l'identité belge reste toujours beaucoup plus forte que l'identité flamande. Selon les études les plus récentes, l'identité belge tend même à se renforcer.

Cette utilisation identitaire est par contre initialement largement absente dans le cas de la télévision francophone, bien qu'elle contribue évidemment aussi structurellement à la constitution d'un espace public francophone en Belgique, puisqu'elle s'adresse spécifiquement à la partie francophone de la population. Mais à ses débuts, une moindre importance est attachée à la télévision, notamment sur le plan de l'information, et on ne retrouve pas de projet politique clair à son égard. Les premières années, c'est même le journal télévisé français qui est retransmis sur la chaîne publique, la RTB («Radio télévision belge »). Plus tard la RTBF - le «F » qui précise le caractère francophone de la chaîne publique est ajouté beaucoup plus tôt que le « $\mathrm{N}$ » de son équivalent flamand - va être confrontée à la dualité intra-francophone incarnée par la coexistence de la communauté française et de la région wallonne. Des nationalistes wallons, comme José Happart, accuseront la RTBF de ne pas soutenir le projet wallon et de se révéler trop bruxello-centriste.

Bien sûr tout cela se passe dans un cadre belge. Et donc, malgré ce que je viens d'expliquer, les médias flamands et francophones véhiculent tout de même également une identité belge. C'est par exemple le cas lorsqu'ils retransmettent les matchs de l'équipe nationale pendant des Coupes du Monde de football. Ces moments d'antenne sont les émissions les plus regardées des deux côtés de la frontière linguistique. C’est aussi le cas lors du couronnement d'un roi ou lors de la fête nationale. Les émissions d'information participent également à la construction d'une identité belge à cause du cadre politique national. Toutefois, il ne s'agit pas de l'émanation d'une volonté ou d'un projet politique, certainement pas du côté flamand.

\section{Concrètement, comment les médias belges participent-ils à modeler certaines identités?}

DS : Etant donné que la plupart des études existantes s'étaient concentrées sur le rôle des médias dans la construction d'une identité propre, d'un " nous », dans ma thèse de doctorat, j'ai surtout analysé comment ces médias construisent une identité de l'« autre», les deux étant toujours intimement liés. Dans le cas belge, l'objectif était alors de déterminer la représentation des francophones véhiculée par les médias flamands et la représentation des flamands véhiculée par les médias francophones. Je me suis concentré sur les émissions informatives à la télévision, comme les journaux télévisés et les débats, diffusés entre 2003 et 2007. Dans ces émissions, j'ai pu observer différentes dynamiques à l'œuvre. J'ai notamment constaté une tendance très forte à l'homogénéisation, l'autre communauté étant représentée comme un bloc homogène, 
par des termes comme " la Flandre », « les flamands », « Wallonië », « de franstaligen », etc. Régulièrement, les déclarations d'un seul responsable politique de l'autre communauté sont assimilées aux vues de l'ensemble des «flamands » ou des "franstaligen ", surtout quand ces déclarations émanent d'une figure considérée comme emblématique d'une communauté. Comme quand, par exemple, les médias flamands parlaient d'Elio Di Rupo - alors président du Parti socialiste - et assimilaient ses propos à l'opinion de l'ensemble des francophones. Dans les médias francophones, c'était surtout la tendance nationaliste qui était attribuée à toute la Flandre. Lors de mes travaux, j'ai donc pu constater qu'une position politique spécifique était attribuée non seulement à l'ensemble de la classe politique de «l'autre " groupe linguistique, mais également à l'entièreté de la communauté formée par les personnes vivant de "l'autre » côté de la frontière linguistique.

On observe aussi une tendance à la différenciation : les médias se focalisent fortement sur les dissemblances entre communautés. Un jour, la VRT a commandé un sondage. La plupart des résultats faisaient état de différences minimes entre les réponses données par les flamands et les francophones. Mais le cadre, le «frame », dans lequel les médias ont présenté ces résultats était celui d'un fossé entre les communautés. Ainsi, une différence de $6 \%$ entre les réponses données par les flamands et les wallons sur une question relative aux radars automatiques était résumée par la formule : "Sire, il n'y a plus de Belges sur les routes». En fait, au moment du sondage, cette question soulevait un conflit communautaire et politique. Et apparemment, c'est à travers le prisme de ce conflit que les médias ont analysé l'opinion publique, en mettant en avant des différences pourtant quasi inexistantes. En période de crises, on assiste aussi à la conflictualisation et à la dramatisation des relations entre les communautés.

Dans les contenus médiatiques, j'ai aussi retrouvé des traces de minorisation et de victimisation : l'autre communauté étant représentée comme celle qui a le dessus et qui domine la communauté du média en question. Ainsi, lorsque Rudy Demotte a accordé une interview à la VRT, du temps où il était ministre fédéral de la Santé, il a été accusé d'avoir pris des mesures défavorables aux flamands. Lors d'une interview accordée à la RTBF sur les mêmes mesures, il lui a été reproché l'inverse, à savoir de prendre des décisions hostiles à l'égard des francophones.

Qu'est-ce que cela nous apprend sur le rôle des médias dans les relations communautaires et le contexte fédéral complexe de la Belgique?

DS : J'ai aussi regardé plus en détail comment les médias traitent la politique fédérale. Ce que j'ai observé peut poser question d'un point de vue démocratique. J'ai notamment identifié les ministres fédéraux auxquels les journaux télévisés donnent la parole. Il faut rappeler que le gouvernement est composé de façon linguistiquement paritaire mais que les ministres prennent des décisions qui ont un impact sur la vie de 
tous les habitants du pays. Quand on regarde les chaînes flamandes - VRT, la télévision publique flamande, et VTM, la plus importante télévision flamande privée -, 80\% des ministres fédéraux qui apparaissent à l'écran sont néerlandophones. Quand on zappe vers la RTBF, la télévision publique belge francophone, et RTL, la télévision francophone privée la plus importante, entre 70 et $80 \%$ d'entre eux sont francophones. Pour ces chaînes, la valeur informative des propos tenus par les ministres fédéraux semble donc dépendre du rôle linguistique de ces derniers. Et ce n'est pas une question de connaissance de la langue, car des ministres parfaitement bilingues demeurent interviewés plus fréquemment sur les chaînes de leur propre communauté. Par ailleurs, dans un journal télévisé, on pourrait parfaitement sous-titrer ou doubler les entretiens.

J'ai alors analysé un peu plus en profondeur comment cette dynamique fonctionne exactement. Parfois, lorsqu'un thème identique est traité, les médias donnent la parole aux ministres issus de leur propre communauté linguistique. Par exemple, sur le budget, on interviewera soit le ministre du Budget soir celui des Finances étant donné qu'ils sont souvent issus de groupes linguistiques différents. Mais j'ai aussi constaté que parfois, certains sujets politiques ne sont pas traités du tout, quand ils renvoient à la compétence d'un ministre de l'autre communauté.

Comment les médias se positionnent-ils alors dans des conflits communautaires qui opposent les responsables politiques des deux grandes communautés?

DS : J'ai étudié la façon dont les télévisions publiques traitaient d'un tel conflit, notamment la scission de l'arrondissement électoral de Bruxelles-Hal-Vilvorde (BHV), un conflit linguistique qui a été au centre de la longue crise institutionnelle en Belgique : la plupart des responsables politiques flamands voulaient scinder BHV, la plupart des responsables francophones s'y opposaient. J'ai notamment analysé les débats télévisés diffusés par la RTBF et la VRT au cours de la première période durant laquelle ce dossier a été mis à l'agenda. Cela m’a frappé de constater que, malgré le temps d'antenne très important dévoué à ce sujet, les spectateurs francophones et néerlandophones n'ont presque jamais pu assister à un débat sur le fond présentant tant les arguments des responsables politiques francophones que ceux des responsables néerlandophones.

Tout d'abord, et c'était surtout le cas pour les débats à la télévision flamande, le nombre de représentants politiques de «l'autre » communauté linguistique était très limité, ce qui a eu pour conséquence d'accorder quantitativement peu d'attention à leur position. Mais plus fondamentalement, le nœud du problème - BHV, doit-il être scindé ou non ? - n'était jamais au centre du débat. Les discussions partaient d'une prémisse donnée, radicalement différente dans les émissions diffusées du côté néerlandophone et francophone : la nécessité de scinder BHV n'était jamais discutée sur le plateau de De Zevende Dag, le débat dominical de la télévision publique néerlando- 
phone, alors que la nécessité de s'opposer à une scission n'était jamais remise en question à Mise au Point, son équivalent francophone. En d'autres termes, sur les deux plateaux, le débat se déroulait dans le cadre de paramètres définis par le consensus politique de la communauté à laquelle appartient le média. Les journalistes qui animent ce genre de débats confrontent toujours les (rares) responsables politiques invités de l' « autre » communauté au point de vue de «leur » communauté, mais jamais le contraire.

Les débats ont dès lors surtout porté sur des questions concernant les stratégies à mettre en œuvre pour obtenir ou bloquer la scission (selon la chaîne que l'on regarde). Les responsables politiques ont souvent été mis sous pression pour expliquer pourquoi ils n'avaient pas encore réalisé leurs intentions ou promesses sur la question. Quant à savoir si la scission devait être obtenue ou bloquée, j’ai constaté une réciprocité de perspectives entre journalistes et responsables politiques d'une même communauté.

Parfois, cela s'est également traduit au niveau lexical. L'utilisation de références déictiques comme «nous » et « eux » par les journalistes les amenaient à prendre position (et à inciter les téléspectateurs à faire de même). De plus, le conflit était régulièrement représenté à travers des métaphores guerrières dramatisantes, comme lorsque les médias faisaient usage du mot « front » pour désigner l'une des deux positions («le front flamand» et «le front francophone») ou encore lorsqu'ils utilisaient des expressions comme "défendre les francophones », "le maillon faible dans le camp francophone », «la Flandre pourrait plier», «les flamands ne reculent pas », «la riposte face aux revendications flamandes », « l'annexion de territoire flamand », « le fer de lance de la bataille des francophones en périphérie », ... )

Même des éléments factuels ont été accentués ou omis selon qu'ils s'intégraient ou pas dans ce consensus politique. Lors d'émissions télévisées telles que De Zevende Dag, on pouvait entendre des allusions continues à un arrêt important de la Cour constitutionnelle qui aurait stipulé que la scission de BHV devait absolument être réalisée, affirmation toutefois incorrecte. À Mise au Point, l'arrêt - élément important du problème puisqu'il prescrivait que le problème de l'arrondissement électoral devait être réglé - n'était jamais mentionné. Les seules exceptions ont eu lieu lorsque des responsables politiques de l'autre communauté ont été invités sur le plateau. Toutefois, les arguments mis en avant par ces "autres voix » semblaient se heurter à un mur invisible, les empêchant de s'insérer dans le débat.

Il manque donc un authentique débat fédéral sur une question fédérale comme la scission de BHV et, à cet égard, les médias ne jouent pas le rôle que l'on pourrait attendre d'eux en tant que facilitateurs du bon fonctionnement d'une sphère publique fédérale. En outre, ils semblent avoir contribué à la polarisation des positions politiques et à une incompréhension mutuelle sur la question, et par conséquent aussi à la longue crise politique qu'a connue la Belgique récemment. 
Constate-t-on une évolution du comportement des médias, parallèle à l'évolution politique de la Belgique?

DS : En partie, oui. La longue crise politique a bien sûr intensifié les conflits mais a aussi suscité une tendance dans l'autre direction, entre autres à la suite de l'émoi suscité par le fameux "journal-bidon» de la RTBF de décembre 2006, qui relayait l'information selon laquelle le parlement flamand avait proclamé l'indépendance de la Flandre. Un débat modeste sur l'image que donnent les médias de l'autre communauté a alors vu le jour. La sensibilité des journalistes à cet égard a un peu grandi et l'on constate une indifférence moindre sur la question. On a aussi rencontré des initiatives d'échanges et de collaboration entre médias flamands et francophones. Les quotidiens flamand, De Standaard, et francophone, Le Soir, par exemple, ont travaillé sur des projets communs et, depuis, les rédactions politiques collaborent plus souvent sur des questions de politique nationale.

Aujourd'hui, je ne crois pas qu'on organiserait encore facilement des débats télévisés sur des thèmes communautaires sans inviter quelqu'un de l'autre communauté, comme au temps des discussions autour de BHV. Mais cela reste difficile de convaincre certains responsables politiques de venir sur le plateau de l'une ou l'autre chaîne. De plus, on a toujours tendance à homogénéiser. Je le constate personnellement, lorsque des médias m'approchent. Sur des plateaux francophones on me pose régulièrement des questions du type : «Monsieur Sinardet, qu'en pense la Flandre ?». J'ai alors tendance à dire : « si vous voulez le savoir, vous auriez dû inviter la Flandre ». Parce que, généralement, il y a bien sûr différents points de vue en Flandre, comme en Belgique francophone. Les médias flamands me posent d'ailleurs souvent le même genre de question sur la Wallonie. Après avoir fait des analyses de contenus médiatiques, je peux donc maintenant dire que je fais aussi de l'observation participante.

On voit à quel point le contexte, notamment politique, influence la façon dont travaillent les médias. Pourriez-vous expliciter les interdépendances qui modèlent leur rôle?

DS : Le contexte politique et institutionnel au sein duquel les médias opèrent est bien sûr crucial. En effet, notre système fédéral, tout comme notre système médiatique, est basé sur une division profonde des groupes linguistiques. La Belgique est la seule entité fédérale sans partis politiques fédéraux, et même le système électoral veut que les électeurs ne puissent voter que pour les responsables politiques de leur propre communauté linguistique. Cela n'est bien sûr pas sans conséquences sur l'information véhiculée par les médias.

Par exemple, un dimanche midi, il est électoralement plus rentable pour un politicien flamand de se rendre sur un marché local que de participer aux grands débats politiques télévisés de la RTBF ou de RTL-TVI. Il touchera en effet plus d'électeurs par cette première démarche que par la seconde. Pour les responsables politiques - y 
compris pour ceux qui prennent des décisions concernant l'ensemble des citoyens du pays en tant que ministres fédéraux - il est électoralement sans intérêt d'exposer leur vision politique ou d'expliciter leur point de vue dans les médias de l'autre communauté. De leur côté, les journalistes ne seront pas enclins à inviter ces politiciens (sauf si leur poids politique est perçu comme extrêmement important) car ils sont électoralement inintéressants pour leur public. C'est une des raisons pour lesquelles la plupart des débats politiques et d'autres émissions, y compris celles relatives à des thèmes fédéraux, rassemblent des responsables politiques de la même appartenance linguistique. Dans ces conditions, on ne parle pas habituellement avec mais plutôt de l'autre communauté. Et, à nouveau, cela entraîne une information communautaire peu nuancée, assortie de stéréotypes et de caricatures. Les dynamiques centrifuges du système institutionnel et du système médiatique - le second découlant du premier en sortent indiscutablement renforcées.

Cette situation pose aussi un problème du point de vue démocratique. Des ministres fédéraux, qui dirigent la totalité du pays, ne répondent souvent de leur responsabilité que devant «leurs » médias et donc "leur» opinion publique. Lors d'élections fédérales, il n’y a en fait aucun débat fédéral véritable : des représentants des partis politiques flamands et francophones débattent chacun de leur côté, dans leur propres médias, au sujet de la politique qu'ils devront mettre en œuvre, une fois les élections passées, avec les représentants de l'autre communauté. Pour qui veut analyser le rôle des médias dans la problématique communautaire, il convient donc d'être attentif au contexte institutionnel plus large dans lequel opèrent les médias.

\section{Peut-on aller jusqu'à dire que cette situation engendre un déficit démocratique?}

DS : Je crois que oui. En tout cas, si l'on se réfère à la théorie de la sphère publique (public sphere) développée par Jurgen Habermas. Ce dernier considère l'existence de sphères publiques comme un élément indispensable au bon fonctionnement d'une démocratie. Les médias, à travers les espaces de communication publique qu'ils créent, sont porteurs de telles sphères publiques. Ils peuvent informer sur les décisions prises dans une démocratie et être une plate-forme de débats publics où les acteurs pertinents confrontent leurs différentes positions. Ils sont capables de générer un débat public commun, au cours duquel des sujets identiques peuvent être abordés $\mathrm{au}$ même moment et dans les mêmes conditions. La sphère publique ainsi constituée doit aussi permettre d'influencer les preneurs de décisions politiques, de les responsabiliser quant aux choix qu'ils opèrent et de transformer l'opinion publique en pouvoir politique.

Ces dernières décennies, des formes de gouvernance dépassant le cadre traditionnel de la démocratie nationale n'ont cessé de gagner en importance. Des systèmes politiques et des institutions transnationaux sont apparus, comme l'Union européenne. Mais alors même qu'actuellement nous évoluons de plus en plus vers un système de 
" démocratie post-nationale », les sphères publiques restent largement nationales. Elles sont donc devenues de plus en plus inadéquates, en ce sens qu'elles ne permettent ni l'émergence d'un débat démocratique, ni une participation citoyenne au sujet d'un grand nombre de politiques importantes aujourd'hui. C'est pour cela que nombre d'auteurs - comme Habermas - considèrent que l'on ne pourra pallier le déficit démocratique de l'Union européenne qu'avec le développement d'une sphère publique européenne. Cette dernière devrait se faire, si pas sous la forme de vrais médias paneuropéens, au moins à travers l'européanisation des médias nationaux et notamment à travers un traitement similaire de l'information. Ainsi, pour certains sujets, les médias nationaux des Etats de l'Union européenne pourraient donner la parole aux mêmes protagonistes, adopter un angle d'approche similaire et ainsi favoriser un traitement « européen » d'une même information.

Dans le cas d'Etats fédéraux multilingues comme la Belgique, les choses ne sont pas nécessairement simples non plus, comme je viens de l'illustrer par le recours à l'exemple des ministres fédéraux qui ne sont pas présents dans les médias des deux côtés de la frontière linguistique ou en évoquant les débats relatifs à BHV. On ne peut donc pas vraiment parler d'une véritable sphère publique « belge », à travers laquelle les médias fournissent une information objective sur les questions politiques (fédérales), et qui permet aux citoyens de participer au débat public en se forgeant une opinion sur base d'arguments rationnels développant les différentes positions.

Donc, si l'on suit la logique de la public sphere theory, le manque de sphère publique belge signifie que la Belgique connait un déficit démocratique. Cependant, on pourrait aussi dire que le problème réside plutôt dans cette théorie, qui date de la période de domination des Etats-nations et qui ne semble pas adaptée à un monde de plus en plus post-national et multilingue. La Belgique pourrait alors plutôt être vue comme un précurseur de cette réalité qui nous incite à profondément repenser cette théorie et à trouver une réponse à l'une des questions fondamentales d'aujourd'hui : comment faire fonctionner des démocraties multilingues d'une façon démocratiquement légitime? 